\title{
Experimental Investigation of Online Path Planning for Electric Vehicles
}

\author{
Christoph Winter, Peter Ritzer, Jonathan Brembeck
}

\begin{abstract}
This paper describes a real-time capable online path planning on roads and its experimental investigation for the highly maneuverable robotic electric vehicle research platform ROboMObil. The path planning algorithm is based on an efficiently solvable and compact optimization problem and contributes to the autonomous driving of centralized controlled vehicles. The necessary development from a global offline problem formulation towards an online receding horizon method is shown, which is capable of taking environmental changes into account. The online planned path together with a generated velocity profile serves as an input to the ROboMObil's geometric path following control, allowing for automated driving. Finally, a test drive shows the results of implementing the presented algorithms on this research vehicle and investigates the energy saving capabilities of the proposed path planning methodology.
\end{abstract}

\section{INTRODUCTION}

Nowadays, vehicles include a variety of comfort and safety relevant assistance systems, like ABS, ESP and cruise control. This development is currently enhanced leading to an increased number of advanced driver assistance systems with the ultimate goal of autonomous driving. An essential part of autonomous or semi-autonomous control strategies is the motion demand planner, generating control inputs for the vehicle controller. The motion demand planner characterizes the vehicle behavior and hence allows consideration of an important aspect of modern mobility, energy and resources saving locomotion. Not only with respect to increasingly scarce raw materials but also due to the limited range of purely electric vehicles, an energy saving operation is important. The path planning, beside other energy management strategies, is a contribution to the overall energy optimized operation of the vehicle.

In [1] a global offline path planning approach is proposed based on two-dimensional splines, which handles the road boundaries as constraints of the mathematical optimization problem. The cost criterion of the optimization problem is a simplified quadratic formulation of the path curvature. The minimization of the path curvature is claimed to be optimal in terms of string energy of the path, but in [1] only the mathematical cost criterion and not physical energy is evaluated. The path curvature is also part of the optimization

Christoph Winter is research assistant and $\mathrm{PhD}$ student at the DLR Institute of System Dynamics and Control (SR), Oberpfaffenhofen, Germany. (E-mail: Christoph.Winter@dlr.de).

Peter Ritzer is research assistant and PhD student at the DLR Institute SR, Germany (until June 2016).

Jonathan Brembeck is provisional head of the Automotive Vehicle System Dynamics Department in the DLR Institute SR, Germany problem in [2], where a two level approach for online path planning based on Bézier curves is used. In [2] the focus lies on a smooth online planning with collision avoidance and no energy related investigation is carried out. Another online path planning approach in [3] uses a simple vehicle model to determine time-dependent smooth and feasible trajectories. The optimization in [3] takes into account obstacles and the deviation from the road centerline.

This paper focuses on the following two aspects: Firstly, an online path planning (OPP) on roads over a moving horizon is presented, that is based on the curvature minimization problem developed in [4]. The energy consumption of the vehicle is influenced by the path planning and by the followed velocity planning on the path. Because it is very difficult to achieve real-time path and velocity planning on an a priori unknown route in such a way that a given journey time for the whole route is hold, the velocity planning is considered being decoupled from the path planning. In the second part of the paper it is investigated by means of experimental results, whether only the curvature minimization of the path (i.e. the path planning) leads to an overall reduced physical energy consumption of the test vehicle for a fixed velocity planning algorithm.

The OPP presented here is part of DLR's electro-mobility demonstrator ROboMObil [5], see Fig. 1. As the path planning is the top level of the energy management it deals with the largest time-horizon. The underlying faster part is the vehicle dynamics control [6], which has the interface of motion demand to the higher level path planner. The aim of the vehicle dynamics control is to allocate the control variables resulting from the motion demand to the actuators so that a robust and energy optimal control is achieved.

The paper is organized as follows: In Section II the real-time capable path planning formulation is sketched, which is solved by an adapted nonlinear optimization method. Furthermore, the theoretical background of the possible energy saving capabilities of the presented approach is

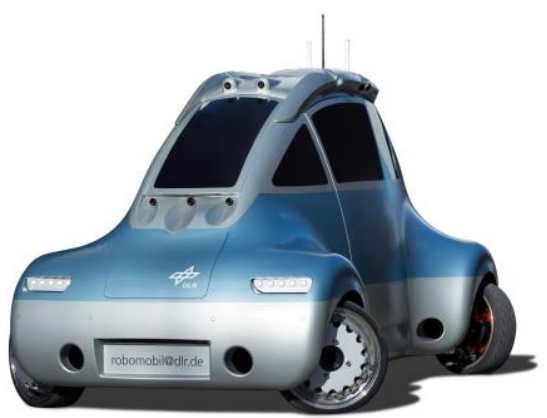

Fig. 1:The DLR ROboMObil [5]. 
discussed. The required developments towards an online path planning algorithm are presented in Section III with a detailed investigation of the optimization horizon. Section IV shows experimental results of the real world test drives with the ROboMObil, determining whether the path optimization leads to a reduced energy consumption. Finally, a short overview of the on-going research is given.

\section{ReAl-Time CAPABle Path PlanNing}

The theoretical background of the energy saving capabilities of the path curvature minimization is presented in Section II.A as a motivation. Furthermore, the optimization problem formulation of the path planning algorithm developed in [4] is repeated in Section II.B for the sake of completeness. The basis of the path planning problem is the situation as shown in Fig. 2. A vehicle is traveling along a predefined road and it is able to use the available lateral space $w_{\text {res }}$ on the street to minimize a certain criterion.

\section{A. Theoretical Background of Energy Saving}

This sections explains the choice of curvature as minimization objective of path planning algorithms, wherefore the conditions are pointed out under which the curvature minimization can lead to an energy saving effect. The energy is not addressed directly as optimization objective, since the coupling between path and velocity planning would not allow a computational fast real-time application. Although there are approaches dealing with velocity optimization within the path planning [7], those approaches are minimizing the error with respect to a given reference velocity instead of generating such a reference. As driving conditions a rural road is considered. In general there are two types of losses in a driving electric vehicle: losses proportional to the velocity, e.g. air drag, and losses proportional to the acceleration, e.g. in the traction motors. With a given maximum velocity and the aim to travel a distance in a certain time, merely the losses due to acceleration can be affected to reduce the overall energy consumption. The slope of the road is not considered in the path planning itself but in the velocity profile generation. Equation (1) shows the power losses of a permanent magnet synchronous machine [8], which is considered as traction motor in an electric vehicle, consisting of ohmic losses, inverter losses and losses from eddy current and hysteresis effects:

$$
P_{\text {loss }}=\underbrace{\frac{3}{2} R i_{q}^{2}}_{\text {ohmic }}+\underbrace{P_{\text {inv, const }}+k_{\text {inv }} i_{q}}_{\text {inverter }}+k_{\text {hyst }} \omega+k_{\text {eddy }} \omega^{2},
$$

with $\omega$ the rotational motor speed, $k$ the respective constant gains of the losses and the current $i_{q}$. The ohmic losses as main part of the power losses are proportional to the acceleration. For that reason the aim is to minimize unnecessary velocity changes in the vehicles path.

The coupling between acceleration $a$, which is indirectly minimized, and the path curvature $\kappa$, which is directly addressed in the optimization problem, is given by the velocity profile generation. The velocity profile generation is based on [9] and can act as a reference for motion control. The generation of the velocity profile consists of the

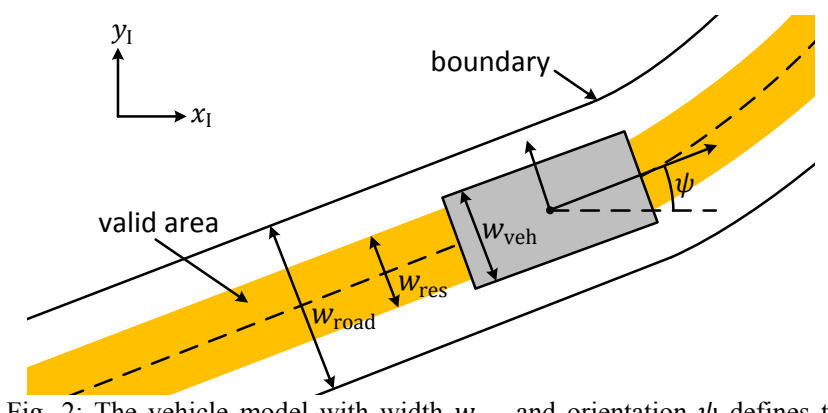

Fig. 2: The vehicle model with width $w_{\text {veh }}$ and orientation $\psi$ defines the valid area of the road (graphic is based on [1]).

following main steps as depicted in Fig. 3. In the first step a desired velocity profile $v$ considering the maximum vehicle velocity and legal speed limits is modified to satisfy a lateral acceleration limit $a_{\text {lat,max }}$ with the given path curvature $\kappa$ regarding the following equation:

$$
v \leq \sqrt{\frac{a_{\text {lat,max }}}{\kappa}}
$$

The second step is a forward and backward filtering of the velocity profile to fulfill the longitudinal acceleration limits of the vehicle. In conclusion, with a smaller curvature of the path and a fixed lateral acceleration limit, a turn can be passed with a higher velocity according to (2) and thus the energy losses due to acceleration of the vehicle are reduced. This motivates the choice of path curvature as optimization objective besides a higher driving comfort.

\section{B. Mathematical Path Representation}

The mathematical path representation described in this section leads to a small scale optimization problem with linear constraints. The path representation uses twodimensional cubic splines consisting of $n-1$ polynomials $\boldsymbol{o}_{k}(q)$ between $n$ interpolation points as path representation as in [1] with spline parameter $q \in \mathbb{R}$ and interpolation points $q_{k} \in \mathbb{N}$ as given in:

$$
\boldsymbol{o}_{k}(q)=\boldsymbol{a}_{k}\left(q-q_{k}\right)^{3}+\boldsymbol{b}_{k}\left(q-q_{k}\right)^{2}+\boldsymbol{c}_{k}\left(q-q_{k}\right)+\boldsymbol{d}_{k} .
$$

The interpolation points $\boldsymbol{o}_{k}\left(q_{k}\right)$ can be moved on a line between the boundary nodes $\boldsymbol{l}\left(q_{k}\right)$ and $\boldsymbol{r}\left(q_{k}\right)$ in dependency of the scalar scaling factor $\alpha_{k} \in[0,1]$ as proposed in [10] and depicted in Fig. 4. This linear relationship is given by
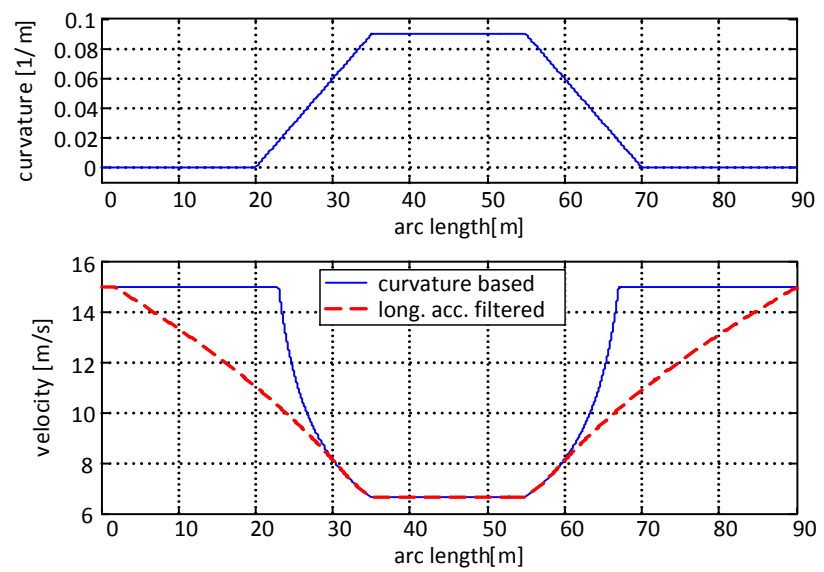

Fig. 3: The curvature of the test track results in the curvature based velocity profile (solid blue line) that fulfills the lateral acceleration limits. The filtering (dashed red line) ensures the longitudinal acceleration limits. 


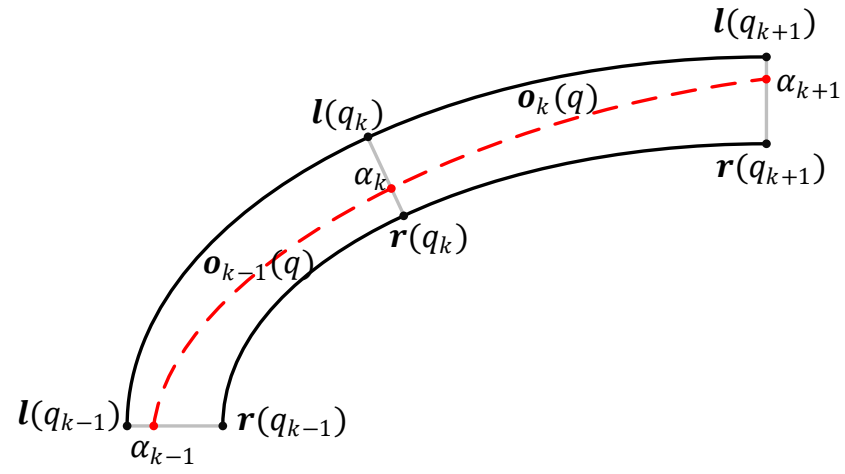

Fig. 4: Notation of the spline representation with the spline $\boldsymbol{o}_{k}(q)$ being shifted between the boundary nodes $\boldsymbol{l}\left(q_{k}\right)$ and $\boldsymbol{r}\left(q_{k}\right)$ by $\alpha_{k}$.

$$
\boldsymbol{o}_{k}\left(q_{k}\right)=\boldsymbol{l}\left(q_{k}\right)+\alpha_{k}\left(\boldsymbol{r}\left(q_{k}\right)-\boldsymbol{l}\left(q_{k}\right)\right) .
$$

Thus the number of optimization variables per spline segment can be reduced from eight polynomial coefficients to only three. The reduced set of variables for one spline segment $\boldsymbol{o}_{k}(q)$ are the two second degree polynomial coefficients $\boldsymbol{b}_{k}=\left(b_{k, x} b_{k, y}\right)$ and $\alpha_{k}$. All other spline parameters $\boldsymbol{a}_{k}, \boldsymbol{c}_{k}, \boldsymbol{d}_{k}$ can be expressed in dependency on those variables [4].

In summary an optimization problem with a nonlinear cost function and linear constraints is proposed. As cost function the quadratic path curvature $\kappa^{2}$ is chosen as used in many other path planning problems [1], [2], [10] and motivated in Section II.A. Thus the potentially energy saving capabilities of the curvature minimization can be investigated in the results in Section IV.B. The equality constraints of the optimization problem ensure a smooth transition between the spline segments and the inequality constraints keep the path within the road boundaries, described by splines $\boldsymbol{l}(q)$ and $\boldsymbol{r}(q)$. The optimization problem with the reduced set of spline coefficients $\boldsymbol{x}=\left(b_{1, x}, b_{1, y}, \alpha_{1}, \ldots, b_{n, x}, b_{n, y}, \alpha_{n}\right)^{T}$ is given as:

$$
\begin{aligned}
\boldsymbol{x}^{*}=\underset{\boldsymbol{x}}{\operatorname{argmin}} & \int_{0}^{q_{n}} \kappa^{2}(\boldsymbol{x}, q) \mathrm{d} q \\
\text { s.t. } & \boldsymbol{A} \boldsymbol{x}=\boldsymbol{b} \\
& \boldsymbol{C} \boldsymbol{x} \leq \boldsymbol{d} .
\end{aligned}
$$

The artificial spline coefficients $\boldsymbol{b}_{n}$ are required to give the spline a defined boundary condition at the end. A real-time capable nonlinear gradient method similar to [11] is chosen as optimization algorithm to solve the minimization problem.

\section{ONLINE PATH PLANNING}

The developments towards a reactive online path planning approach to deal with environmental changes are discussed in this section. In [4] the required mathematical path representation is proposed (see Section II.B) and this paper completes the online planning approach with an investigation of the horizon and the start position determination. The main idea of the OPP is a continuous sequence of optimized path segments over a certain horizon. Thereby the following scenario is considered: While driving along a road the part of the road ahead of the vehicle is optimized like described in Section II.B including the $n-1$ polynomials. This part of the road is called a segment. In time step $i$ the vehicle drives along the previously optimized path segment while the next segment is optimized. The positioning of those segments along the road moves with the vehicle and time (see Section III.B).

The continuous transition of position, orientation and curvature of the spline between the previously and currently planned path segments is essential to guarantee an overall smooth path and a high driving comfort. This transition is enabled by the spline reformulation as described in [4] which allows incorporating the curvature as additional boundary condition at the beginning of the path segment. Without this reformulation only the position and orientation of the spline could be matched at the transition between two segments. This transition between two segments should not be confused with the transition between two polynomials of the spline, which is continuous due to the equality constraints of the optimization problem. The other important steps, like finding the correct start position and an appropriate horizon size, are presented in the following subsections.

\section{A. Boundary Definition and Start Position Determination}

In this work the XML-based road description OpenDrive [12] is used to determine the required road information. The open format specification is able to describe entire road networks but here the focus lies on the road information of the individual tracks. A road segment is defined according to a reference line parameterized by arc length $s_{\mathrm{r}}$. The OpenDrive road description serves as a function determining the reference line position $\boldsymbol{p}_{\mathrm{r}}\left(s_{\mathrm{r}}\right)$ together with the orientation $\varphi\left(s_{\mathrm{r}}\right)$ of the reference line and the track width in dependency of a given arc length $s_{\mathrm{r}}$. Thus the boundary positions $\boldsymbol{l}\left(s_{\mathrm{r}}\right)$ and $\boldsymbol{r}\left(s_{\mathrm{r}}\right)$ for the optimization constraints can be obtained by simple geometric relations based on the road description, possibly augmented by other information source like vision based sensors. From those boundary positions $\boldsymbol{l}\left(s_{\mathrm{r}}\right)$ and $\boldsymbol{r}\left(s_{\mathrm{r}}\right), n$ for each site, the splines $\boldsymbol{l}(q)$ and $\boldsymbol{r}(q)$, required for the inequality constraints in (5), are interpolated. These boundary splines are approximating the road boundaries.

The introduced road definition is parameterized over arc length $S_{\mathrm{r}}$. Because in general the arc length of the road $S_{\mathrm{r}}$ differs from the optimized paths arc length $s_{0}$, it is important to distinguish between these two arc lengths. For a seamless transition between two path segments it is necessary to start the current optimization in time step $i$ with the position $\boldsymbol{o}_{1}(q=0)$ on the previously in time step $i-1$ optimized path segment at point $\boldsymbol{p}_{\mathrm{o}}$. This point $\boldsymbol{p}_{\mathrm{o}}$ lies on the path a certain distance ahead of the vehicle, depending on the horizon size of the path planning. The alignment of $\boldsymbol{p}_{\mathrm{r}}\left(s_{\mathrm{r}}^{*}\right)$ and $\boldsymbol{p}_{\mathrm{o}}$ is required to align the boundary points $\boldsymbol{l}\left(s_{\mathrm{r}}\right)$ and $\boldsymbol{r}\left(s_{\mathrm{r}}\right)$ with the optimization starting point $\boldsymbol{p}_{\mathrm{o}}$, which is given by the previous planned path. The boundary points are depending on the road description at $\boldsymbol{p}_{\mathrm{r}}\left(s_{\mathrm{r}}\right)$, as described before. Only with this alignment the problem formulation of Section II.A can be applied with the optimization variable $\alpha$ shifting the optimized path points $\boldsymbol{o}_{k}\left(q_{k}\right)$ between the 


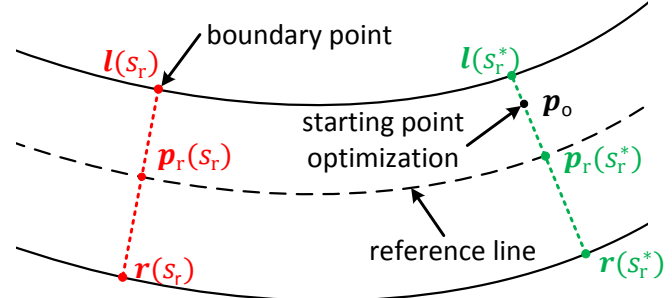

Fig. 5: Determination of arc length $s_{\mathrm{r}}^{*}$ so that the starting point of the optimization $\boldsymbol{p}_{\mathrm{o}}$ lies on the same line as the boundary points $\boldsymbol{l}\left(s_{\mathrm{r}}^{*}\right)$ and $\boldsymbol{r}\left(s_{\mathrm{r}}^{*}\right)$.

boundary points. Fig. 5 shows the correctly aligned positions at $S_{\mathrm{r}}^{*}$ marked green and another position marked red at which no meaningful shifting regarding (4) would be possible. The alignment depicted in Fig. 5 is equal to the calculation of $S_{\mathrm{r}}^{*}$ so that the reference line position $\boldsymbol{p}_{\mathrm{r}}\left(s_{\mathrm{r}}^{*}\right)$ has a minimum distance to the point $\boldsymbol{p}_{\mathrm{o}}$ of the previous optimized path segment [13] as given in:

$$
s_{\mathrm{r}}^{*}=\underset{\mathrm{s}_{\mathrm{r}}}{\operatorname{argmin}}\left\|\boldsymbol{p}_{\mathrm{r}}\left(s_{\mathrm{r}}\right)-\boldsymbol{p}_{\mathrm{o}}\right\|_{2}^{2} .
$$

With $\boldsymbol{p}_{\mathrm{r}}\left(s_{\mathrm{r}}^{*}\right)$ at a minimum distance to $\boldsymbol{p}_{\mathrm{o}}$, the point $\boldsymbol{p}_{\mathrm{o}}$ is located on the normal line of the road at $\boldsymbol{p}_{\mathrm{r}}\left(s_{\mathrm{r}}^{*}\right)$ and therefore on the same line as the boundary points, as required for the shifting by $\alpha$. The distance minimization in (6) can be transferred to an equivalent root finding problem as stated in (7), derived from the analytical solution of (6)

$$
\underbrace{\boldsymbol{e}\left(s_{\mathrm{r}}\right)^{T} \cdot \boldsymbol{t}_{\mathrm{r}}\left(s_{\mathrm{r}}\right)}_{e_{\text {long }}} \stackrel{!}{=} 0 .
$$

As depicted in Fig. 6, $\boldsymbol{e}\left(s_{\mathrm{r}}\right)$ is the position error between $\boldsymbol{p}_{\mathrm{r}}\left(s_{\mathrm{r}}\right)$ and $\boldsymbol{p}_{\mathrm{o}}$. Furthermore, $\boldsymbol{t}_{\mathrm{r}}\left(s_{\mathrm{r}}\right)$ is the tangential vector to the reference line at $\boldsymbol{p}_{\mathrm{r}}\left(s_{\mathrm{r}}\right)$. The condition of (7) is that the error vector and the tangential vector are perpendicular to each other or equivalently the longitudinal error $e_{\text {long, }}$, which is marked yellow in Fig. 6, is zero at $\boldsymbol{p}_{\mathrm{r}}\left(s_{\mathrm{r}}^{*}\right)$. For this problem it is stated in [14] that $\boldsymbol{p}_{\mathrm{r}}\left(s_{\mathrm{r}}^{*}\right)$ exists and is unique, if $\left\|\boldsymbol{e}\left(s_{\mathrm{r}}^{*}\right)\right\|_{2}$ is smaller than the lower bound of the curve radii. The root finding problem (7) can be solved by the iteration law (8) in iteration step $m+1$ based on [13]:

$$
s_{\mathrm{r}}(m+1)=s_{\mathrm{r}}(m)+K_{P} \cdot e_{\text {long }}(m) .
$$

The arc length at the next iteration step $s_{\mathrm{r}}(m+1)$ is derived from the arc length at the $m$-th step $s_{\mathrm{r}}(m)$ and the longitudinal error $e_{\text {long }}(m)$. From multiple tests it turned out that the proportional gain $K_{P}=1$ is a good choice. The start value of the iterative approach is computed as

$$
s_{\mathrm{r}, i}(m=0)=s_{\mathrm{r}, i-1}^{*}+\Delta s_{\mathrm{c}}
$$

in the $i$-th time step regarding the piecewise path planning. The term $\Delta s_{\mathrm{c}}$ determines the driven path length of the vehicle during one time step. By shifting the last optimal arc

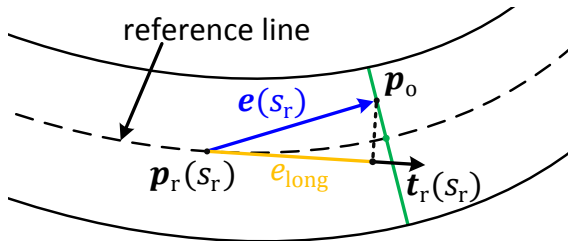

Fig. 6: Finding $\boldsymbol{p}_{\mathrm{r}}\left(s_{\mathrm{r}}\right)$ nearest to $\boldsymbol{p}_{\mathrm{o}}$ by minimizing $e_{\text {long. }}$. The optimum $\boldsymbol{p}_{\mathrm{r}}\left(s_{\mathrm{r}}^{*}\right)$ with zero longitudinal error is marked with a green line. length $s_{\mathrm{r}, i-1}^{*}$ by the currently covered distance $\Delta s_{\mathrm{c}}$ a growing displacement of the start value can be avoided.

\section{B. Path Segmentation}

The aim of an online receding horizon path planning can only be achieved if the path is planned over a finite spatial horizon. A global offline path planning could not take changes in the environment, resulting in changes of the optimization constraints, into account. The OPP is a stepwise process over a horizon moving with the vehicle. In each step the optimization problem (5) is solved and a fixed reference path for the path following control [13] is generated. This section describes the segmentation of the path in front of the vehicle into parts which are optimized and parts which remain unchanged from the previous time step.

Fig. 7 illustrates the optimization horizon and the path segmentation schematically. The main principle of the approach shown in Fig. 7 is that the vehicle moves along the fixed planned path of the previous time step while the path segment in front is optimized during the current step. The path segment, which is the reference path for the vehicle is marked with a solid red line in Fig. 7 and the path segment, which is optimized is marked with a dashed blue line. While the vehicle is moving on the previously computed fixed path segment $\Delta s_{0}$, the next road segment with a length of $\Delta s_{\mathrm{r}}$ is optimized whereby $\Delta s_{\mathrm{r}}$ must be longer than $\Delta s_{\mathrm{o}}$ to preplan a long enough path segment. At the end of each time step the reference path for the next step is computed (indicated with dashed arrows in Fig. 7). The extended reference path in the $(i+1)$-th time step, shown with the dotted red line in Fig. 7 , consists of the previously generated reference path from $s_{\mathrm{c}, i}$ to $s_{\mathrm{c}, i}+\Delta s_{\mathrm{o}}$ and the subsequent optimized path from $s_{\mathrm{c}, i}+\Delta s_{\mathrm{o}}$ to $s_{\mathrm{c}, i}+2 \Delta s_{\mathrm{o}}$. Thereby $s_{\mathrm{c}, i}$ states the actual driven arc length positions of the vehicle at the beginning of time step $i$. This extended reference path is longer than necessary, including also unused parts, e.g. behind the vehicle. It covers both extreme cases: On the one hand, the case that the vehicle does not move from time step $i$ to $i+1$, i.e. $s_{\mathrm{c}, i+1}=s_{\mathrm{c}, i}$ and the reference path with a length of $\Delta s_{\mathrm{o}}$ reaches from $s_{\mathrm{c}, i}$ to $s_{\mathrm{c}, i}+\Delta s_{\mathrm{o}}$ as already in time step $i$. On the other hand, the case that the vehicle moves with maximum velocity, i.e. $s_{\mathrm{c}, i+1}=s_{\mathrm{c}, i}+\Delta s_{\mathrm{o}}$ and the reference path reaches from $s_{\mathrm{c}, i}+\Delta s_{\mathrm{o}}$ to $s_{\mathrm{c}, i}+2 \Delta s_{\mathrm{o}}$. The part of the extended reference path actually used by the vehicle in time

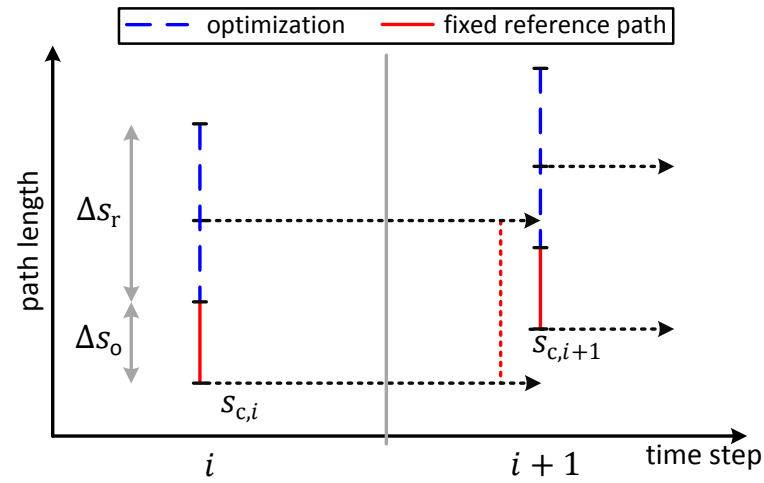

Fig. 7: Overview of optimization horizon and path segmentation. 
step $i+1$ is indicated by the solid red line in Fig. 7, starting from the actually vehicle position $s_{c, i+1}$. The use of the extended reference path is required, since the position $s_{\mathrm{c}, i+1}$ is not known at the end of time step $i$ when the reference path of time step $i+1$ is generated. Hence, only variables known at the beginning of the time step $i$, i.e $s_{\mathrm{c}, i}$ and the horizon size are used to define the reference path.

\section{Size of Optimization Horizon}

In the previous section it is assumed that the vehicle drives along a path with a length of $\Delta s_{0}$ during one time step. In the same time a road segment with a horizon length $\Delta s_{\mathrm{r}}$ is optimized. This section describes how the lengths $\Delta s_{0}$ and $\Delta s_{\mathrm{r}}$ are derived. The requirement on the length of the reference path $\Delta s_{0}$ is to be longer than the distance driven by the car during one time step. Equivalently (10) describes this condition in the time domain

$$
t_{\mathrm{opt}} \stackrel{!}{<} t_{\text {drive }}=\frac{\Delta s_{\mathrm{o}}}{v_{\max }}
$$

with $t_{\mathrm{opt}}$ the computation time of the optimization and $t_{\text {drive }}$ the lower bound of the driving time of the vehicle along a path with a length of $\Delta s_{0}$. The time $t_{\mathrm{opt}}$ is given by the realtime implementation resulting in a lower bound of the reference path length $\Delta s_{0}>t_{\mathrm{opt}} \cdot v_{\max }$.

For the derivation of a lower bound of the horizon size $\Delta s_{\mathrm{r}}$ two requirements has to be considered. The first obvious requirement is $\Delta s_{\mathrm{r}}>\Delta s_{0}$, as can be seen in Fig. 7, since the fixed path segment $\Delta s_{\mathrm{o}}$ is part of the road segment with a length of $\Delta s_{\mathrm{r}}$ which is optimized. The second requirement additionally considers the velocity profile generation. To ensure the vehicle is always able to come safely to a full stop, zero velocity at the end of the optimized path is demanded. As a consequence a deceleration phase at the end of the velocity profile is always required. If the horizon $\Delta s_{\mathrm{r}}$ is too short an unwanted sawtooth shaped profile can result from the deceleration, like shown in Fig. 8 and described subsequently. Considering a straight road, the desired velocity of the vehicle is equal to its maximum velocity $v_{\text {max }}$. At the end of each planned velocity profile for one path segment of the online approach, indicated by the different colors and line types in Fig. 8, the desired velocity is reduced to zero. If the reference path with a length of $\Delta s_{\mathrm{o}}$ of the optimized path segment reaches this deceleration region the start velocity of the next time step is lower than $v_{\text {max }}$. As a consequence in the next time step the velocity is first increased due to a desired velocity of $v_{\max }$ and then decreased due to a safe deceleration at the end. The resulting overall reference path is a sawtooth shaped velocity

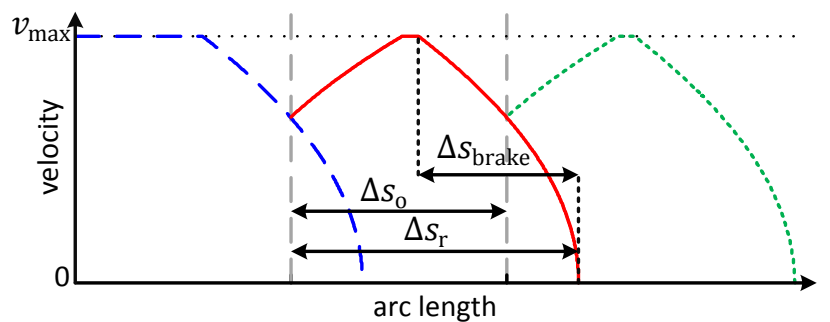

Fig. 8: The horizon $\Delta s_{\mathrm{r}}$ must be long enough, such that the braking profile is not contained in the reference path. profile although a constant velocity of $v_{\max }$ is suitable. This yields the following further condition on the horizon

$$
\Delta s_{\mathrm{r}}>\Delta s_{\mathrm{o}}+\Delta s_{\text {brake }},
$$

with $\Delta s_{\text {brake }}=-v_{\max }^{2} / 2 a_{\min }$ the distance required by the vehicle to constantly decelerate with the minimum acceleration $a_{\min }$ from maximum to zero velocity. In equation (11) ensures that the deceleration phase is not included in the fixed reference path.

\section{EXPERIMENTAL INVESTIGATION}

This section shows the results of implementing the presented online path planning approach based on curvature minimization on the research vehicle ROboMObil. An investigation of the energy saving capabilities of the proposed methodology in real world test drives is carried out. The OPP module is implemented in MATLAB/Simulink beside the vehicle dynamics control and path following control [13] functions and then compiled for a real-time rapid prototyping platform.

\section{A. Analyses of measured Signals}

The analysis of the energy consumption in Section IV.B is based on the following evaluation of the sensor logging signals of the ROboMObil. The main energy consumption comes from the four individual permanent magnet synchronous machines (PMSM) of the traction motors and the four PMSM steering motors. The energy consumption of other peripheral devices is constant and not affected by the type of driven path and therefore it is neglected in the energy analysis.

The overall power consumption of the actuators consists of two main parts: the electrical losses $P_{\text {loss }}$ of the PMSM as formulated in (1) and the mechanical power $P_{\text {mech }}$ at time $t$

$$
P_{\text {mech }}(t)=\omega(t) \cdot\left(\tau(t)+\tau_{\text {fric }}\right),
$$

resulting from the rotational speed $\omega$, the applied torque $\tau$ and additional friction losses with a constant friction torque $\tau_{\text {fric }}$. Finally, the overall energy consumption $E$ is computed as $E=\int P_{\text {mech }}(t)+P_{\text {loss }}(t) \mathrm{d} t$. All required sensor signals, such as motor torque, motor speed, path parameters and the vehicle position, are recorded with a sample time of $4 \mathrm{~ms}$. It is not possible to directly use the battery current and voltage for the computation of the overall power consumption of the vehicle, due to a very slow sample time of the battery management system and the corresponding logging signals.

\section{B. Test Drive Results}

The results of the test drives on two different tracks with the ROboMObil utilizing the OPP and the path following control are discussed in this section. First of all, Fig. 9 gives an overview of the online planned path on the first test track and the respective power consumption of the actuators. The coloring illustrates where power is consumed and where it is recuperated. The evaluation of the overall energy consumption of the actuators required to drive three laps of the optimized path and three laps in the middle of the test track (no optimization), acting as a reference, is shown in TABLE 1 . The required energy is clearly reduced by $19 \%$ 

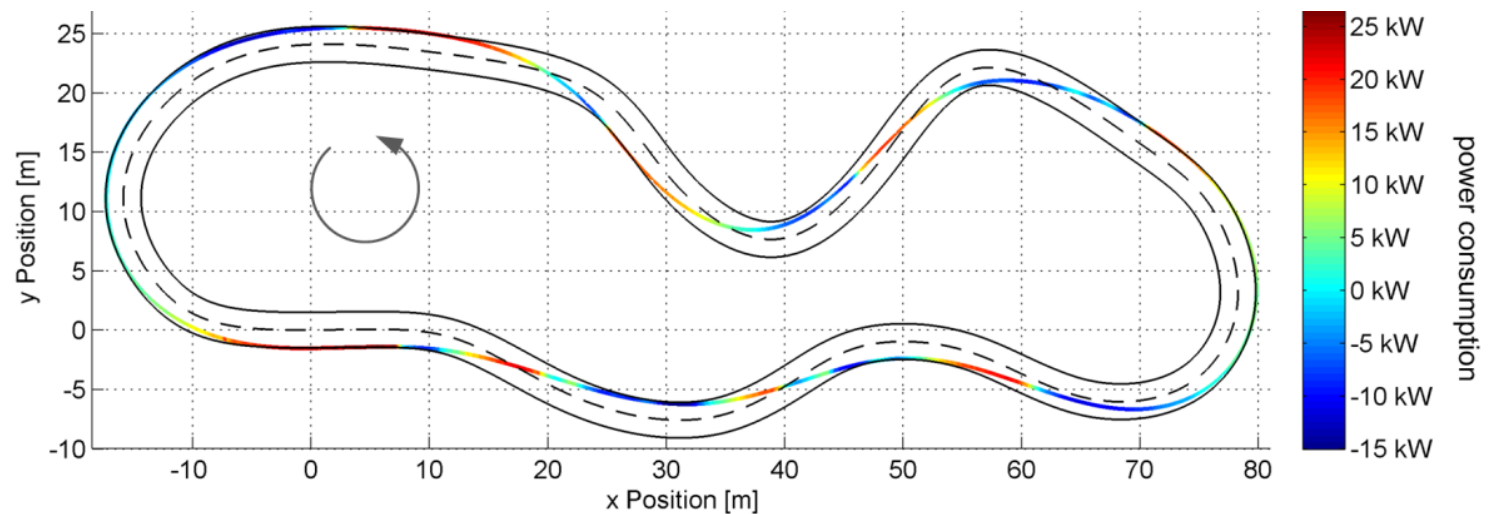

Fig. 9: Online optimized path with visualisation of the actuator's power consumption on the first test track (driving direction counterclockwise).

by the OPP although the drive time is also reduced by $10 \%$, which demonstrates the energy saving capabilities on this track. For both paths the same parameters of the velocity profile generation are used.

TABLE 1: Energy comparison between the reference and the online optimized path with a horizon of $100 \mathrm{~m}$ and width of $3 \mathrm{~m}$.

\begin{tabular}{cccc}
\hline \hline Path & Energy & Drive Time & Energy Saving \\
\hline Reference & $549 \mathrm{~kJ}$ & $111.8 \mathrm{~s}$ & - \\
Online Optimized & $445 \mathrm{~kJ}$ & $101.3 \mathrm{~s}$ & $19 \%$ \\
\hline
\end{tabular}

As second part of the results, a further test drive on a second test track on very limited space, as shown in Fig. 10, is discussed. The slightly deviation of the measured vehicle position beyond the boundaries results from the proportional control error of the lateral position controller of the path following control and the high lateral acceleration on this track. The second test drive provides an impression of the limitations of the curvature minimization approach, dealing solely with the path planning without considering the velocity planning [15] directly. On the second test track the energy consumed while driving along the curvature optimized path is $3.7 \%$ higher than on the reference path. An explanation for the slightly higher energy consumption gives the comparison of the velocity profile for both paths, optimized and non-optimized, in Fig. 11. On the one hand, the velocity of the optimized path is almost always higher than the velocity of the reference path resulting in a $6.5 \mathrm{~s}$ shorter driving time for one lap on the $150 \mathrm{~m}$ long track. This consistently higher kinetic energy leads to a higher

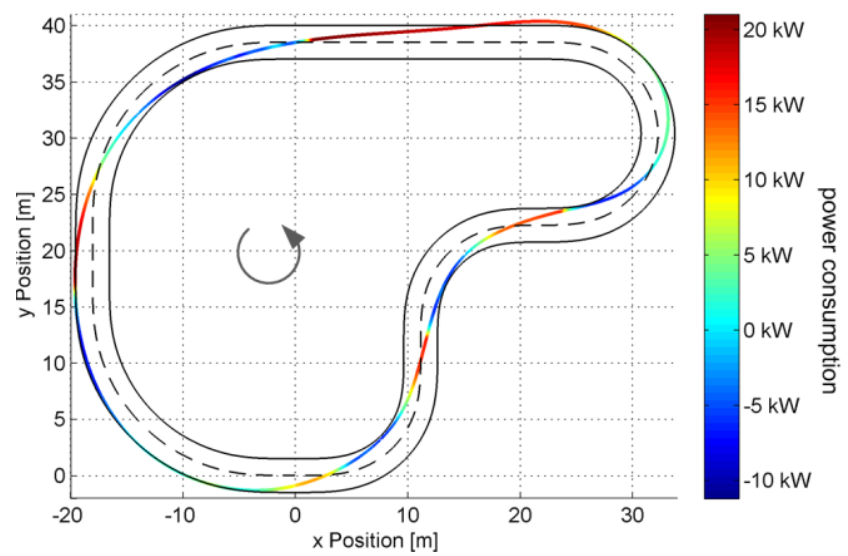

Fig. 10: Online optimized path with visualisation of the actuator's power consumption on the second test track (driving direction counterclockwise). overall energy consumption. On the other hand, the maximum velocity of $10 \mathrm{~m} / \mathrm{s}$ for this track is never reached, which is in contrast to the assumption of a more constant velocity interrupted by deceleration phases due to a high curvature of the path, as it is the condition from Section II.A. The velocity profile of the online planned path has slightly longer acceleration phases, higher rotational speeds of the motors and a higher air drag. As a result no savings in the power losses are achieved on the second track.

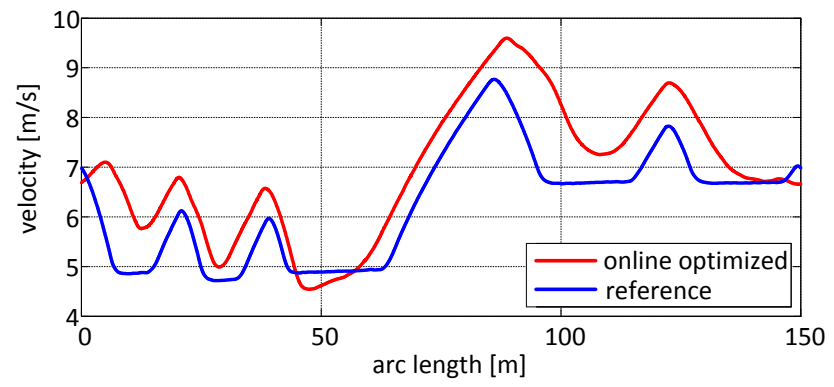

Fig. 11: Velocity profile of the online optimized path and the reference path of the second test track.

The OPP with its piecewise manner combined with the velocity profile generation cannot incorporate the driving time as a constraint for higher comparability between reference and optimized path. Even in a global optimization approach the coupling between path and velocity planning under a time constraint would not be trivial. However, for both paths the toolchain is identically showing the difference in the energy consumption based on the path planning.

\section{Discussion AND OUTLOOK}

The results demonstrate the potential of the curvature based online path planning, over a moving horizon, for saving energy while travelling along a given road using an electric vehicle like the ROboMObil. Nevertheless, the experimental investigation shows that the curvature as optimization criterion not always leads to a reduced energy consumption. As the path planning indirectly influences the energy consumption through the velocity profile, ongoing research has to deal with an extension to directly address the velocity as part of the path planning optimization problem. The presented curvature based OPP provides a smooth path with high driving comfort and can serve as a good basis for energy optimal velocity planning, because of the reduced 
necessary longitudinal acceleration of the generated path. The moving horizon of the real-time capable online path planning enables the optimization to take environmental changes into account by repositioning the boundaries. It is also planned to investigate the online re-planning capabilities with time variant obstacles along the path.

\section{REFERENCES}

[1] J. Daniel, A. Birouche, J.-P. Lauffenburger and M. Basset, "Navigation-based constrained trajectory generation for advanced driver assistance systems," International Journal of Vehicle Autonomous Systems, vol. 9, no. 3-4, pp. 269-296, 2011.

[2] L. Ma, J. Yang und M. Zhang, „A Two-level Path Planning Method for On-road Autonomous Driving," in International Conference on Intelligent System Design and Engineering Application, 2012.

[3] C. c. Li, I. Wang, X. Wang und Y. Zhang, „A Model based Path Planning Algorithm for Self-driving Cars in Dynamic Environment,“ in Chinese Automation Congress (CAC), 2015.

[4] J. Brembeck and C. Winter, "Real-time capable path planning for energy management systems in future vehicle architectures," in IEEE Intelligent Vehicles Symposium, 2014.

[5] J. Brembeck, L. M. Ho, A. Schaub, C. Satzger, J. Tobolar, J. Bals and G. Hirzinger, "ROMO - The Robotic Electric Vehicle," in 22nd IAVSD International Symposium on Dynamics of Vehicle on Roads and Tracks, 2011.

[6] R. de Castro, T. Bünte and J. Brembeck, "Design and Validation of the Second Generation of the Robomobil's Vehicle Dynamics Controller," in International Symposium on Dynamics of Vehicles on Roads and Tracks (IAVSD), 2015.

[7] J. Ziegler, P. Bender, T. Dang und C. Stiller, „Trajectory planning for Bertha - A local, continuous method," in IEEE Intelligent Vehicles Symposium Proceedings, Dearborn, 2014.

[8] D. Schröder, "Verluste und Erwärmung im Antriebssystem," in Elektrische Antriebe-Grundlagen, Springer Verlag, 2009, pp. 41-72.

[9] T. Bünte and E. Chrisofakis, "A Driver Model for Virtual Drivetrain Endurance Testing," in Proceedings of 8th International Modelica Conference, 2011.

[10] F. Braghin, F. Cheli, S. Melzi and E. Sabbioni, "Race Driver Model," Computers and Structures, vol. 86, no. 13-14, pp. 1503-1516, 2008.

[11] J. Rosen, "The Gradient Projection Method for Nonlinear Programming. Part I. Linear Constraints," Journal of the Society for Industrial and Applied Mathematics, vol. 8, no. 1, pp. 181-217, 1960.

[12] M. Dupuis, "OpenDRIVE Format Specification," 2010. [Online]. Available: http://www.opendrive.org/docs/OpenDRIVEFormatSpecRev1.3D.pdf. [Accessed 09 June 2016].

[13] P. Ritzer, C. Winter and J. Brembeck, "Advanced path following control of an overactuated robotic vehicle," in IEEE Intelligent Vehicles Symposium, 2015.

[14] P. Morin and C. Samson, "Motion Control of Wheeled Mobile Robots," in Handbook of Robotics, Springer, 2008, pp. 799-826.

[15] T. v. Keulen, B. d. Jager, D. Foster und M. Steinbuch, „Velocity Trajectory Optimization in Hybrid Electric Trucks, “ in American Control Conference, Marriott Waterfront, Baltimore, MD, USA, 2010. 